\title{
Cellular Complexity of Hemochorial Placenta: Stem Cell Populations, Insights from scRNA-seq, and SARS-CoV-2 Susceptibility
}

\author{
Christopher S. Mallery Jr. ${ }^{1,5} \cdot$ Maira Carrillo ${ }^{1,2} \cdot$ Ariel Mei $^{1,3} \cdot$ Ana Correia-Branco ${ }^{1} \cdot$ Olga Kashpur $^{1}$. \\ Mary C. Wallingford ${ }^{1,4}$ (i)
}

Accepted: 15 July 2021 / Published online: 20 October 2021

(c) The Author(s), under exclusive licence to Springer Nature Switzerland AG 2021

\begin{abstract}
Purpose of Review The placenta is a transient organ that forms de novo and serves a critical role in supporting fetal growth and development. Placental oxygen, nutrients, and waste are transported through processes that depend on vascular structure and cell type-specific expression and localization of membrane transporters. Understanding how the placenta develops holds great significance for maternal-fetal medicine. The purpose of this review is to examine current information regarding placental progenitor populations.

Recent Findings Recent advancements in single-cell RNA sequencing (scRNA-seq) provide unprecedented depth for the investigation of cell type-specific gene expression patterns in the placenta. Thus far, several mouse placenta scRNA-seq studies have been conducted which produced and analyzed transcriptomes of placental progenitors and cells of the fully developed placenta between embryonic day (E) 7.0 and E12.5. Together with human placenta scRNA-seq data which, in part, has been produced through coordinated research campaigns in the scientific community to understand the potential for SARS-CoV-2 infection, these mammalian studies lend fundamental insight into the cellular and molecular composition of hemochorial placentae found in both mouse and human.

Summary Single-cell placenta research has advanced understanding of tissue-resident stem cells and molecules that are poised to support maternal-fetal communication and nutrient transport. Herein, we provide context for these recent findings by reviewing placental anatomy and cell populations, and discuss recent scRNA-seq mouse placenta findings. Further research is needed to evaluate the utility of placental stem cells in the development of new therapeutic approaches for the treatment of wound healing and disease.
\end{abstract}

Keywords Placenta $\cdot$ Placental development $\cdot$ Placentation $\cdot$ SARS-CoV- $2 \cdot$ Single-cell RNA sequencing $\cdot$ Stem cells

\section{Introduction}

This article is part of the Topical Collection on Stem Cell Switches and Regulators

Mary C. Wallingford

mwallingford@tuftsmedicalcenter.org

1 Tufts Medical Center, Mother Infant Research Institute, 800 Washington St, Boston, MA 02111, USA

2 Odessa College, $201 \mathrm{~W}$ University Blvd, Odessa, TX 79764, USA

3 Simmons University, 300 Fenway, Boston, MA 02115, USA

4 Division of Obstetrics and Gynecology, Tufts University School of Medicine, 800 Washington Street, Boston, MA 02111, USA

5 Texas A\&M University - San Antonio, One University Way, San Antonio 78224, USA
The placenta performs multiple functions that are essential for fetal development and the maintenance of maternal health during pregnancy. It is a transient organ across which oxygen, carbon dioxide, water, nutrients, and waste are transported between maternal and fetal circulations. The placenta also produces hormones and angiogenic molecules that participate in maternal adaptations to pregnancy. The mouse placenta is a hemochorial-type placenta, characterized by two distinct circulations, maternal-placental (uteroplacental) and fetal-placental (fetoplacental), and the direct interaction of maternal blood with chorionic epithelial cells of extraembryonic origin [1]. Genes that are critical for placentation and placental nutrient transport have been discovered through human genomics, agricultural cloning, and mouse genetics. 
The mouse has been particularly critical in understanding genetic pathways that regulate differentiation of placental progenitors, as it is a widely used model of mammalian genetics. Thousands of knockout mouse strains have been generated and characterized by individual investigators as well as newer coordinated efforts, such as those driven by the Knock Out Mouse Project (KOMP) [2], and international phenotyping efforts coordinated by Deciphering the Mechanisms of Developmental Disorders Programme (DMDD) [3, 4], and the International Mouse Phenotyping Consortium (IMPC) [5, 6]. The mouse model enables the collection and analysis of placental progenitors and placenta tissue across all stages of pregnancy through the use of timed mating and precise microdissection. To date, an immense knowledge base has been constructed that serves as a foundational understanding of the processes and molecular players that give rise to diverse cell types in the hemochorial placentae, including trophoblast stem cells and tissueresident stem cells. More recently, the advent of single-cell RNA sequencing (scRNA-seq) has begun to build on this foundation by uncovered complex networks and previously unrecognized diversity and plasticity in placental cell populations which we are just beginning to understand. In the following sections, we summarize placental anatomy and developmental milestones, review the current understanding of hematopoietic competency of the placenta, summarize recent mouse placenta scRNA-seq studies, and discuss recent human placenta single-cell studies analyzed through coordinated efforts to determine the potential for vertical transmission of SARS-CoV-2. Together, these recent mouse and human studies have significantly expanded the scientific understanding of the complex molecular and cellular composition of the hemochorial placenta.

\section{Foundations of Placental Development: a Brief Overview of Tissues, Cells, Molecules, and Epigenetics}

The mouse placenta contains multiple compartments, including the remodeled decidua, the junctional zone, the labyrinth, and the chorionic plate (Fig. 1a, d). The labyrinth of the placenta harbors a specialized maternal-fetal interface (MFI) which mediates maternal-fetal communication. Oxygen, nutrients, and waste are transported across the MFI through processes that depend, in part, on the structure and

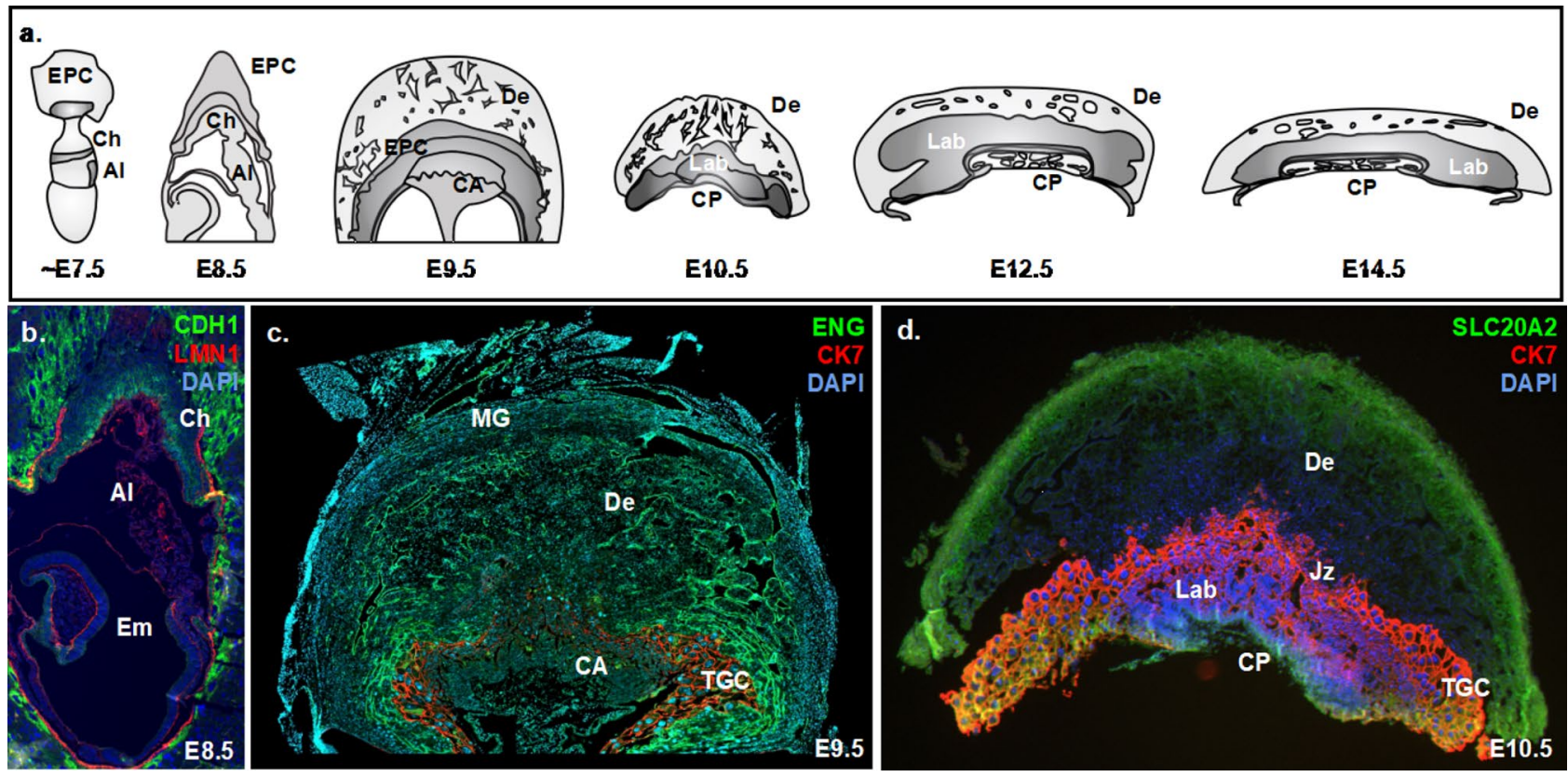

Fig. 1 Placental tissues analyzed through scRNA-seq or snRNA-seq studies. Tissues that have been analyzed through scRNA-seq span E7.0-E14.5 (a). These include embryonic and extraembryonic tissues isolated from the gastrulating mouse embryo between E7.0 and E7.75, as well as from Theiler stage 12 embryos at E8.5. After chorioallantoic fusion, available single-cell studies have focused on the chorioallantois (developing labyrinth), labyrinth, ectoplacental cone, and chorionic plate. Examples of formalin-fixed paraffinembedded (FFPE) E8.5, E9.5, and E10.5 mouse placenta tissue sections stained with immunofluorescent antibodies are shown (b-d).
Immunofluorescent labels include antibodies against the epithelial marker e-cadherin (CDH1), the basal lamina protein lamininalpha1 (LMN1), the endothelial protein endoglin (ENG), the trophoblast marker cytokeratin 7 (CK7), and the placental phosphate transporter SLC20A2. Abbreviations: Al, allantois; CA, chorioallantois; $\mathrm{Ch}$, chorion; CP, chorionic plate; De, decidua; E, embryonic day; EPC, ectoplacental cone; EXE, extraembryonic ectoderm; Jz, junctional zone; Lab, labyrinth; MG, metrial gland; Mm, Mus musculus; TGC, trophoblast giant cells; TS, Theiler stage 
surface area of maternal-placental and placental-fetal vascular compartments and cell type-specific expression and localization of membrane transporters. Understanding how the specialized vasculature of the MFI develops and functions holds great significance for maternal-fetal medicine. The side of the MFI that interacts with fetal blood is lined by endothelial cells of extraembryonic origin, and the side of the MFI that interacts with maternal blood is uniquely lined by cells of the trophoblast lineage $[7,8 . \bullet \bullet]$.

Trophoblasts are indeed a major cell type in the placenta, and numerous subtypes of trophoblasts have been described that perform diverse yet essential functions. Trophoblasts are derived from trophoblast stem cells that first appear during preimplantation development. This early cell lineage division occurs at embryonic day (E) 3.5 in the mouse, at the blastocyst stage which is characterized by the presence of an inner cell mass (ICM), a blastocoel cavity, and a well-defined distinct outer trophectoderm (TE). At the molecular level, the trophoblast lineage presents specific hypomethylation of the Elf5 promoter [9] and can be identified by the expression of $C d x 2$ [10], a class I homeobox transcription factor. The specification of embryonic and TE lineages is initiated by transcriptional networks in which OCT4 (POU5F1) and CDX2 play central roles [10-12]. In the TE, $C d x 2$ expression is activated in part by HIPPO signaling and restricted to the outer cells upon compaction of the morula at $\sim \mathrm{E} 2.5$, resulting in a commitment to the TE lineage [10-13]. In both human and mouse, CDX2/Cdx2, TEAD4/Tead4, and OCT4/Oct4 transcripts are enriched in the blastocyst, and loss of function mouse studies have demonstrated their roles in TE and ICM cell fate determination, respectively $[14,15]$.

The TE gives rise to epithelial trophoblast lineages of the placenta (embryonic ectoderm and ectoplacental cone), whereas the ICM is generally considered to give rise to the embryo proper, with some exceptions. By E6.5, the mouse blastocyst has developed into an egg cylinder morphology, and the tissues that will give rise to fetal membranes and placenta have formed, including the amnion, chorion, and allantois [11]. The development of the labyrinth starts at $\sim$ E8.5 with the fusion of chorionic mesothelium, extraembryonic ectoderm (ExE), and allantoic mesoderm, as diagrammed in Fig. 1a, b. By E9.5, allantoic vessels invaginate towards chorionic folds, and the primitive interhemal membrane forms which contains syncytiotrophoblast cells, mesenchyme, basal lamina, and endothelial cells (Fig. 1a, c). As development progresses, the placental labyrinth becomes increasingly convoluted into an intricate labyrinthine structure (Fig. 1a-d). The MFI is fully formed by $\sim$ E12.5 in the mouse. The fully developed mouse MFI contains three trophoblast layers: non-syncytial sinusoidal trophoblast giant cells (S-TGC) and two layers of syncytiotrophoblast cells (SynT-I and SynT-II). SynT-I is proximal to the maternal blood space, and SynT-II is juxtaposed to the basal lamina of fetal capillaries. SynT-I cells are MctI and Syna positive, whereas SynT-II expresses Gcml, Cebpa, and Synb [16]. Outside of the labyrinth, trophoblast giant cells (TGCs) are in direct contact with the maternal decidua. Lastly, spongiotrophoblast (SpT) and glycogen cells (GC) form the major endocrine compartment of the placenta $[17,18]$. Together, these trophoblast subtypes perform the central functions of the mouse placenta, which are to provide oxygen and nutrients to the fetus, while removing carbon dioxide and other waste products, to metabolize substances and hormones, and to protect the fetus against infections and xenobiotics.

In addition to gene expression changes and mRNA or protein markers, divergent DNA methylation patterns have been observed in embryonic and extraembryonic compartments that are critical to the commitment of lineage-specific differentiation and development $[19,20 . \bullet \bullet$. The de novo DNA methylation patterns are mediated by DNA methyltransferases (DNMT), including DNMT3A, DNMT3B,

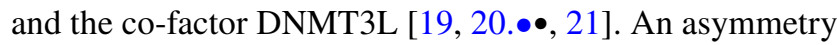
between embryo and placenta DNA methylation patterns occurs rapidly during preimplantation development, initiating a de novo methylation wave between E4.5 and E7.5. The expression of Dnmt3b is higher than Dnmt $3 a$ in the epiblast and embryonic derived cells, whereas relative expression of Dnmt $3 b$ is reduced in the ExE and trophoblast lineage [22]. At E6.5, the epiblast presents a higher DNA methylation state in comparison to the ExE [22]. Recent studies by Legault et al. support that DNMT3B activity is initially the main provider of this asymmetric methylation mark at the peri-implantation stage, while DNMT3A becomes the principal de novo methyltransferase as development progresses [23]. In the fully developed placenta, methylation profiles in retrotransposons Rltr 10 and Rltr 20 also display heterogeneity between placental layers in the mouse, particularly in the labyrinth across which maternal-fetal transport of nutrients and oxygen occurs, and the endocrine junctional zone [24]. A future investigation employing multi-omics studies that integrate this mRNA, protein, and epigenetic data is needed to better understand tissue-wide mechanisms of gene expression and stem cell maintenance in the placenta.

\section{Tissue-Resident Placental Stem Cells in the Placenta: Hemogenic Endothelium and Mesenchymal Stem Cells}

The previous section describes progenitor cells observed during early development of the placenta, yet a large body of research supports that the placenta also harbors tissueresident stem cells that have the potential to differentiate into multiple cell types when cultured ex vivo, including cells with hematopoietic potential and mesenchymal stem cells. 
Developmentally, the establishment and onset of hematopoiesis occur in unison with the development of vascular networks during embryogenesis. Briefly, hematopoiesis occurs in two waves termed primitive hematopoiesis and definitive hematopoiesis, which are described in depth in a recent review by Jagannathan-Bogdan and Zon [25]. In the mouse model, primitive hematopoiesis initiates in blood islands at embryonic day (E) 7.25 [26]. The yolk sac hemogenic endothelium is the first to give rise to erythrocytes and macrophages during primitive hematopoiesis [25]. Cells produced in the primitive wave are poised to provide oxygen to the embryonic tissues prior to definitive hematopoiesis and blood flow [25, 26]. Definitive hematopoiesis gives rise to hematopoietic stem cells (HSCs) and multi-lineage hematopoietic stem and progenitor cells (HSPCs) that differentiate and subsequently migrate to multiple locations during embryonic development and into adulthood, including the placenta and bone marrow [27]. Erythrocytes that form during primitive hematopoiesis differ from those produced by definitive hematopoiesis and are larger and nucleated. Definitive hematopoiesis is observed at $\sim$ E8.25 in the yolk sac, $\sim$ E9.5 in the placenta, and in the aorta-gonad-mesonephros (AGM) at $\sim$ E10.5. Hematopoiesis within the AGM peaks at around E10.5, at which time HSPCs and HSCs are generated and begin to migrate to the fetal liver ( $\sim \mathrm{E} 11.0)$, before residing in the bone marrow at E16.5 [26, 28-31].

HSCs are derived from hemogenic endothelial cells, a subset of specialized tissue-resident endothelial cells that have the potential in situ to give rise to HSCs and downstream HSPCs in vivo and in vitro [26, 29, 32, 33]. At the molecular level, mouse HSCs are characterized by the presence of SCA1, c-KIT, CD31, and integrin alpha 2b (CD41) proteins [34-36]. HSC can be detected within chorioallantoic mesenchyme and fetal vessels of the placental labyrinth during midgestation in the mouse [36]. They are first observed at $\sim$ E9.0 in the mouse placenta and increase dramatically in number between E12.0 and E13.0 [30, 34-36]. The first placental HSC clusters originate from the allantois and are regulated by endothelial niche cells [34]. CD41 and c-KIT proteins are both detected in the mouse allantois [37] and, when isolated prior to their fusion, both the chorion and allantois harbor hematopoietic stem cells with the potential to give rise to myeloid and definitive erythroid cells [7, 35, 37, 38]. Overall, these data support that the mouse placenta harbors intrinsic hematopoietic potential.

In addition to hemogenic endothelium and HSCs studied in mouse and human, mesenchymal stromal cells (MSC) are an additional type of tissue-resident placental stem cell which are of great interest for cell-based therapies for the treatment of a variety of disorders including wound healing. MSCs in the human placenta have been studied extensively.
Human MSCs are fibroblast-like cells which have been shown to have multi-lineage differentiation potential and immunomodulatory properties [39, 40]. The Mesenchymal and Tissue Stem Cell Committee of the International Society for Cellular Therapy has defined three MSC criteria: the MSCs must adhere to plastic, express specific surface antigens, and show multipotent differentiation potential [41, 42]. MSC antigens specifically include CD105, CD73, and CD90, and exclude CD45, CD34, CD14/CD11b, CD79a/ CD19, and HLA class II [42]. Currently, four regions of the fetal placenta have been identified as harboring potential stem/progenitor cells, including human amniotic epithelial cells (hAECs), human amniotic mesenchymal stromal cells (hAMSCs), human chorionic mesenchymal stromal cells (hCMSCs), and human chorionic trophoblastic cells (hCTCs) [41, 43]. In addition, MSC have been isolated from chorionic villi and maternal decidua basalis as well as the umbilical cord (Wharton's jelly) [43]. Finally, a subset of trophoblasts have also been shown to lack expression of MHC class II antigens which normally mediate graft rejection [44]. The lack of MHC class II antigens supports that MSC are strong candidates for the cell-based treatment of disease, including wound healing, but further research is needed to further characterize the molecular composition and plasticity of these cells. Importantly, scRNA-seq provides a new avenue for in-depth and high-throughput understanding of MSCs and may provide critical stepping stones for translating tissue-resident placental stem cells into the clinic.

\section{Single-Cell Analyses of Mouse Placenta: Recent Studies of Placental Progenitors and the Cellular Composition of the Fully Developed Placenta}

The advent of scRNA-seq has established a new frontier in biomedical research, enabling investigators to detect and describe the breadth of cell type-specific transcriptomes in complex organisms and tissues. A number of groups have already applied scRNA-seq to the analysis of mouse and human placenta. In this section, we discuss the first available scRNA-seq mouse placenta studies which were published between 2016 and 2020. The mouse strain, cell selection method, developmental stage, isolated tissues, sorting and sequencing method, reference genome, and annotation are compared in Table 1. Mouse strains in which placental cells or placental progenitors (EXE and allantois) were examined include CD-1, C57B1/6, and CBA, and the developmental

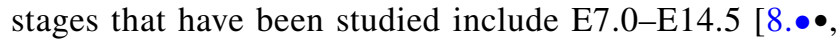

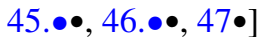

Nelson et al. and Scialdone et al. published the first two scRNA-seq studies to incorporate placental progenitors in 
Table 1 Placental tissues analyzed through scRNA-seq or snRNA-seq studies. Published single-cell or single-nuclei studies are listed which analyze placenta and/or placental progenitor populations. For each study, the mouse strain, cell selection method, developmental stage, isolated tissues, sorting and sequencing method, reference genome, and annotation are compared. The colored dots included next to each citation correspond to tissues diagrammed in Fig. 1a. Angled yellow and blue lines on the E9.5 placenta indicate that this tissue was exam-

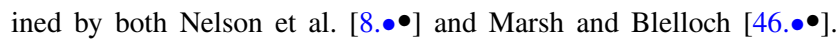
Abbreviations: $C A$, chorioallantois; $C P$, chorionic plate; $E$, embryonic day; $E P C$, ectoplacental cone; $E X E$, extraembryonic ectoderm; $s c R N A$-seq, single-cell RNA sequencing; snRNA-seq, single-nuclei RNA sequencing. Cell selection proteins include integrin alpha chain 2b (CD41), fetal liver kinase 1 (Flk1), and PR domain zinc finger protein $(\operatorname{Prdm} 1)$

\begin{tabular}{|c|c|c|c|c|c|c|c|}
\hline Citation & Strain & Cell Selection & Developmental Stage & Tissus Collected & Sorting and Sequencing & Ref. Genome & Annotation \\
\hline Scialdone et al. 2016 & $\mathrm{CD}-1$ & CD41+, Flk1+ & E7.0 (Primitive streak) & Embryo, EXE & scRNA-seq, SMART-seq2, Manual & M. musculus & Ensembl v38.77 \\
\hline Scialdone et al. 2016 & CD-1 & CD41+, Flk1+ & E7.5 (Neural plate) & Embryo, EXE & scRNA-seq, SMART-seq2, Manual & M. musculus & Ensembl v38.77 \\
\hline Scialdone et al. 2016 & $\mathrm{CD}-1$ & CD41+, Flk1+ & E7.75 (Head fold) & Embryo, EXE & scRNA-seq, SMART-seq2, Manual & M. musculus & Ensembl v38.77 \\
\hline Ibarra-Soria et al. 2018 & C57BL/6, CBA & N/A & $\mathrm{E} 8.25$ (TS12, 2-4s) & Embryo, EXE & scRNA-seq, 10X Genomics & GRCm38.p4 & Ensembl v84 \\
\hline Nelson et al. 2016 & CD-1 & Prdm1-Venus+ & E9.5 & CA, EPC & scRNA-seq, Manual sorting & Mm9 & Ensembl v67 \\
\hline Marsh and Blelloch 2020 & C57BL/6J & N/A & E9.5 & Labyrinth, CP & snRNA-seq, 10x Genomics & Mm10 & CellRanger 3.0.2 \\
\hline Marsh and Blelloch 2020 & C57BL/6J & $\mathrm{N} / \mathrm{A}$ & E10.5 & Labyrinth, CP & snRNA-seq, 10x Genomics & Mm10 & CellRanger 3.0.2 \\
\hline Marsh and Blelloch 2020 & C57BL/6J & N/A & E12.5 & Labyrinth, $\mathrm{CP}$ & snRNA-seq, 10x Genomics & $\mathrm{Mm} 10$ & CellRanger 3.0 .2 \\
\hline Marsh and Blelloch 2020 & C57BL/6J & N/A & E14.5 & Labyrinth, CP & snRNA-seq, 10x Genomics & Mm10 & CellRanger 3.0.2 \\
\hline
\end{tabular}

2016, and both studies utilized mechanical dissociation $[8 . \bullet \bullet, 45 . \bullet \bullet]$. Nelson et al. applied scRNA-seq to examine the function of PRDM1/BLIMP1. A previously validated Prdm1-Venus fluorescent BAC transgenic reporter was employed to enable manual cell selection of individual E9.5

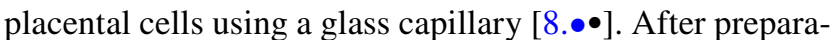
tion and controlling for outliers, the cells were sequenced using an Illumina HiSeq 2500. Based on the expression of 15,402 genes, the investigators identified six distinct populations of cells among 78 samples through the use of hierarchical clustering and principal component analysis, including two of maternal origin and four extraembryonic

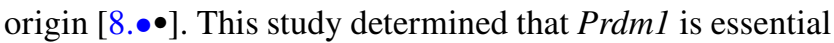
for the specification of spiral artery trophoblast giant cells

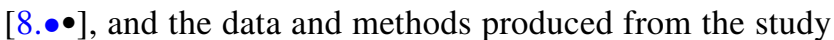
continue to inform and guide single-cell studies in the placenta research field. Scialdone et al. applied the Smart-seq2 protocol and manual sorting to study the mouse epiblast and extraembryonic mesoderm during gastrulation between E7.0

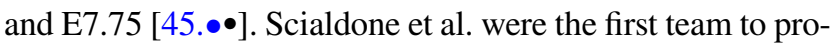
duce a cell type-specific transcriptome-wide in vivo view of early mesoderm formation in a mammalian model [45.••]. This study centered largely on the differentiation of the germ layers and embryonic mesoderm, in particular, and generated novel extraembryonic single-cell data by profiling the cellular composition of the allantois which will give rise to the umbilical cord and fetal endothelial cell compartment of

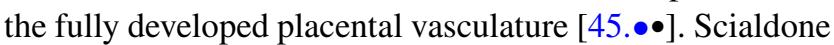
et al. successfully applied scRNA-seq to identify altered distributions of Sox7 and Cbfa2t3, among other genes known to be regulators of blood development [45.••].

In 2018, Ibarra-Soria et al. published whole embryo scRNA-seq findings which characterized E8.25 (late gastrulation) mouse embryonic and extraembryonic cell populations, with a focus on the endodermal, mesodermal, and

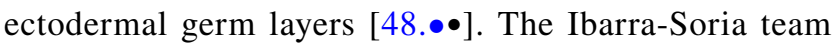
sequenced over 20,000 cells from E8.25 embryos with confirmed Theiler stage 12 (2-4 somite) morphology using the
$10 \times$ Genomic Platform and an Illumina HiSeq 2500 [48.••]. Out of 20,000 cells, transcriptomes for 19,396 cells were analyzed and assigned to 33 distinct clusters by analysis of 15,073 unique transcripts [48.••]. A total of 20 major cell types are described (some encompassing multiple clusters) which expressed a number of germ layer-specific genes, including 869 endodermal genes, 240 mesodermal genes,

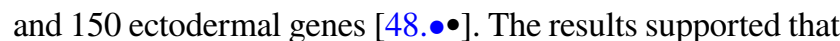
the endothelium is subdivided based on cell maturity and location and in association with the development of blood progenitor cells. In addition to the identification of allantoic and ExE gene clusters, this study revealed a critical role for the leukotriene biosynthesis pathway as a key regulator of

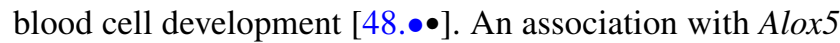
was also observed, indicating that Alox5 regulates leukotriene production and the transition of hemogenic endothelial

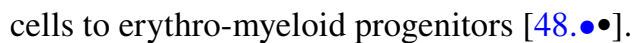

More recently, scRNA has been applied to advance the understanding of cell type-specific transcriptomes in the fully developed mouse placenta [46.••]. Marsh and Blelloch used scRNA-seq to examine the labyrinth in a study published in 2020 that aimed to define the cellular composition of the MFI. This work garnered vast swaths of data on cell trajectories, signaling interactions, and transcriptional drivers that will inform future studies in mouse and human models for years to come [46.••]. Marsh and Blelloch investigated placental scRNA-seq expression profiles at multiple developmental stages following chorioallantoic fusion in order to better understand the differentiation and specialization of chorionic trophoblast in the mouse placental labyrinth, including E9.5, E10.5, E12.5, and E14.5 [46.••]. In contrast to the previously described methods, Marsh and Blelloch employed single nuclei RNA-seq (snRNA-seq) in lieu of scRNA-seq methods which proved incompatible due to the presence of large multinucleated syncytiotrophoblasts in the placenta. Five major cell types were identified by snRNAseq, including decidual stroma, fetal mesenchyme, endothelial cells, blood cells, and trophoblast [46.••]. Although 
decidual stromal cells were largely avoided through excision of the labyrinth between the allantois and the decidua, maternal nuclei were identified by the expression of Xist.

Among the major cell types observed by Marsh and Blelloch, two subgroups of fetal mesenchyme cells were identified, one with pericyte markers, and growth factors associated with vascular development, Wnt5b and $P d g f r b$, and a second population expressing Gata4, Kit, and Pdpn [46.••]. Multiple endothelial cells were also identified, including vascular endothelium (expressing Pecaml, Kdr, and Tek), lymphatic endothelium (Flt4/Vegfr3), a pro-angiogenic lineage (Vegfc), and a lymphogenic lineage (Igfl). Hematopoietic cells included erythrocytes (Hbb-y, Hba-x), macrophages (Mrc1+), B-cells (Bank1, Btla), T-cells (Gzmc, Skamf1, Cd84), and natural killer cells (Cd244) [46.••].

Among the TE lineage, known markers of SynT-I (Tfrc and Mctl) and SynT-II (Epha4, Tgfa, Gcml and Synb) were observed in association as expected. Transporter genes and pathways, in particular, demarcate unique functions for each trophoblast layer. For example, folate receptors Folr1 and Folr2 were detected in association with SynT-I genes, whereas the carboxylate transport genes and cholesterol transporters Scarbl and Vldlr associated with SynTII markers, and Hdlbp and Ldlr were detected in S-TGC, demonstrating the specialization in the function of these trophoblast layers. SpT and GCs of the junctional zone were also identified. Finally, two novel subgroups of labyrinthine trophoblast progenitor cells (LaTP) were observed. Both groups expressed Epcam and differed in part by the level of Met expression. Elevated Met expression was associated with precursors for SynT-II, whereas elevated expression of $T c f 7 l 1$, a transcription factor in the Wnt signaling pathway, was associated with in precursors for SynT-I and S-TGC. In contrast, low Tcf7ll was associated with differentiating SynT-II cells, further supporting the role of Wnt signaling in SynT-II differentiation. Indeed, multiple Wnt signaling genes (e.g., Ror2, $L g r 5$, and $T c f 7 l 1$ ) were upregulated in LaTP and presumptive SynT-II precursors [47•].

Intracellular transport and cell signaling genes consistent with cell-cell communication were also detected in SynTI, SynT-II, and S-TGC cells. The SynT-II transcriptomes revealed increased expression of genes involved in cell-cell and cell-matrix interactions, such as adherens junction proteins and collagens, reflecting the critical barrier function of SynT-II cells which are positioned medially to the SynT-I and fetal endothelium. In contrast, genes involved in signaling and migration were overrepresented in S-TGC, which reside in the maternal blood space and are connected to the SynT-I by highly plastic desmosomes. Cells of a presumptive S-TGC fate also displayed relatively high Lifr expression, consistent with the known requirement for LIF/STAT3 signaling in the normal formation of the labyrinth. LIF/ STAT3 signaling is just one example of a gene that may be important to further understand the origin of placental disorders [49], such as placental abruption, placenta accreta, placenta previa, preeclampsia, and fetal growth restriction, which may be affected by signaling pathways involved in mediating and maintaining the fetal-maternal interface.

\section{Single-Cell Analyses of the Human Placenta: Recent Studies Pertaining to SARS-CoV-2}

As reviewed recently by Li et al., Xiao et al., and Turco and Moffett, scRNA-seq studies of human placenta have generated and expanded understanding of the cellular composition of the human placenta $[50,51 . \bullet, 52]$. For example, the transcription factor gene $\mathrm{Gcml}$ is detected in SynT-II in mice, as well as in intravillous cytotrophoblast cluster 3 in human as identified in Pavlicev et al., supporting a conserved role in syncytiotrophoblast differentiation [53]. As another example of a conserved target which can inform both fundamental understanding of placenta as well as clinical attributes is Plac8. Plac8 is expressed in mouse GCs as well as in human interstitial extravillous trophoblast cells, where it in involved in invasion and migration; Plac8 is upregulated in response to hypoxia and is upregulated in preeclamptic placentas [53, 54]. In contrast, Podxl is highly expressed in mouse S-TGC, which are involved in the formation and maintenance of maternal blood spaces around the interface; meanwhile, Podxl is highly expressed in endometrial stromal fibroblasts in human. These studies in hand with mouse placenta research provide critical information that is needed to understand the level of conservation between mouse and human placenta at the molecular and cellular level. Most recently, these approaches have been employed to better understand the potential for vertical transmission of SARS-CoV-2.

Multiple studies of human scRNA-seq and bulk RNAseq data have been undertaken recently to advance the understanding of placental molecules that may interact with SARS-CoV-2. Early in the start of the SARS-CoV-2 pandemic, Mengmeng et al. used scRNA-seq databases, including the Gene Expression Omnibus (GEO) Database and E-MTAB-6701 to examine the possibility of vertical transmission of SARS-CoV-2 from the mother to the fetus [55]. Human placenta datasets, human fetal heart, liver, and kidney datasets, as well as human and mouse lung datasets, were compiled, analyzed by Seurat computational algorithms, visualized as a violin plot, and statistically evaluated. The angiotensin-converting enzyme 2 (ACE2) receptor for SARS-CoV-2 was indeed heavily expressed in the MFI of mice, supporting that more research was needed to determine if vertical transmission of the virus is possible through this pathway [55], though subsequent studies in human were discordant. In an alternate study, Pique-Regi 
et al. were able to use scRNA-seq to determine that cotranscription of ACE2 and TMPRSS2 is negligible in the placenta and thus concluded that vertical transmission of the virus via this route is unlikely [56]. Accordingly, Mengdie Lü et al. performed single-cell transcriptomic analysis and detected only nine ACE2-positive trophoblasts in the placenta out of 9852, indicating a low possibility of vertical transmission for SARS-CoV-2 [57]. Finally, a recent study by Cui et al. utilized transcriptomes (SMART-Seq2) to demonstrate that the presence of ACE2 and TMPRSS2 is strongly associated with viral invasion, epithelial cell proliferation, and cell adhesion molecule binding [58]. Overall, this body of work suggests that vertical transmission of the SARS-CoV-2 by ACE2 and TMPRSS2 is possible, but rare, and to date, there is still no consensus. Indeed, clinical studies only support rare instances of vertical transmission of SARS-CoV-2, and most reported neonatal infections lack evidence of viral RNA in the fetal blood and amniotic fluid. A recent study by Colson et al. ${ }^{59}$ analyzed the placentas of 31 COVID-19-positive mothers and detected one case of placental infection. The authors further expanded the study with tractable in vitro experiments and did not detect the virus inside primary cytotrophoblasts or syncytiotrophoblasts exposed to SARS-CoV-2 virions. Lastly, the authors did not detect TMPRSS2 in the syncytium, thus supporting that trophoblasts are unlikely to be infected by SARS-CoV-2 at term. Further study is needed to evaluate temporally specific windows of susceptibility to vertical transmission, as well as long-term sequalae that may associate with maternal, fetal, or neonatal SARS-CoV-2 infection.

\section{Conclusions}

The application of mouse placenta scRNA-seq has shown that this methodology and analysis pipeline is a valuable discovery tool for identifying and characterizing cell types without prior knowledge of all cellular phenotypes present within the tissue being examined. scRNA-seq has also been used effectively to test hypotheses surrounding mechanisms of development and disease through the analysis of genetically modified mouse models and clinical tissues. These critical studies build on an already strong foundational knowledge base, yet the complexity that has been discovered through scRNA-seq supports a premise for further, nuanced inquiry into conserved functions of proteins and signaling pathways at the hemochorial maternal-fetal interface of mouse and human. Ultimately, the heterogeneity observed clinically in human placental disorders may also relate, in part, to subtle differences in cellular composition or anatomical position of critical low-number cell populations which may be overlooked in both bulk and single-cell approaches. Looking forward, longitudinal studies which incorporate positional (histological) information and multi-omics data, such as miRNA profiles (which may negatively regulate translation), and proteomes (which provide definitive support of protein abundance), are needed for a systemic spatiotemporal understanding of the placenta across gestation. In conclusion, placenta research at the single-cell level in both mouse and human holds great potential for impact with regard to both advancing fundamental understanding of the MFI and translating this knowledge into novel approaches for diagnosing, monitoring, and treating placental disorders as well as a disorder such as a wound healing which may benefit from placenta cell-based therapies.

Acknowledgements The Wallingford Lab is supported by the National Institutes of Health K99/R00HD090198 (M. Wallingford) and the American Heart Association 19CDA34660038 (M. Wallingford). The authors thank Karen Hirschi and Hunter Wallingford for the discussion that supported the development of this manuscript.

\section{Declarations}

Conflict of Interest The authors declare that they have no conflict of interest.

Human and Animal Rights and Informed Consent This article does not contain any studies with human or animal subjects performed by any of the authors.

\section{References}

\section{Papers of particular interest, published recently, have been highlighted as: \\ - Of importance}

1. Soares MJ, Varberg KM, Iqbal K. Hemochorial placentation: development, function, and adaptations. Biol Reprod. 2018. https://doi.org/10.1093/biolre/ioy049.

2. Dickinson M, et al. High-throughput discovery of novel developmental phenotypes. Nature. 2016;537:508-14.

3. Perez-Garcia V, et al. Placentation defects are highly prevalent in embryonic lethal mouse mutants. Nature. 2018;555:463-8.

4. Mohun T, et al. Deciphering the mechanisms of developmental disorders (DMDD): a new programme for phenotyping embryonic lethal mice. DMM Disease Models and Mechanisms. 2013;6:562-6.

5. Ryder E, et al. Molecular characterization of mutant mouse strains generated from the EUCOMM/KOMP-CSD ES cell resource. Mamm Genome. 2013;24:286-94.

6. Skarnes WC, et al. A conditional knockout resource for the genome-wide study of mouse gene function. Nature. 2011;474:337-44.

7. Azevedo Portilho N, Tavares Guedes P, Croy BA, Pelajo-Machado M. Localization of transient immature hematopoietic cells to two distinct, potential niches in the developing mouse placenta. Placenta. 2016;47.

8. Nelson AC, Mould AW, Bikoff EK, Robertson EJ. Single-cell RNA-seq reveals cell type-specific transcriptional signatures at the maternal-foetal interface during pregnancy. Nat. Commun. 2016;7. Nelson et al. is recognized for its importance as the first mouse placenta scRNA-seq study. 
9. Lee CQE, et al. What Is Trophoblast? A combination of criteria define human first-trimester trophoblast. Stem Cell Reports. 2016. https://doi.org/10.1016/j.stemcr.2016.01.006.

10. Niwa $\mathrm{H}$, et al. Interaction between Oct $3 / 4$ and $\mathrm{Cdx} 2$ determines trophectoderm differentiation. Cell. 2005. https://doi.org/10. 1016/j.cell.2005.08.040.

11. Arnold SJ, Robertson EJ. Making a commitment: cell lineage allocation and axis patterning in the early mouse embryo. Nat Rev Mol Cell Biol. 2009. https://doi.org/10.1038/nrm2618.

12. Rossant J, Tam PPL. Blastocyst lineage formation, early embryonic asymmetries and axis patterning in the mouse. Development. 2009. https://doi.org/10.1242/dev.017178.

13. Strumpf $\mathrm{D}$, et al. $\mathrm{Cdx} 2$ is required for correct cell fate specification and differentiation of trophectoderm in the mouse blastocyst. Development. 2005. https://doi.org/10.1242/dev.01801.

14. Blakeley P, et al. Defining the three cell lineages of the human blastocyst by single-cell RNA-seq. Dev. 2015. https://doi.org/10. 1242/dev.123547.

15. Marikawa Y, Alarcón VB. Establishment of trophectoderm and inner cell mass lineages in the mouse embryo. Mol Reprod Dev. 2009. https://doi.org/10.1002/mrd.21057.

16. Simmons DG, et al. Early patterning of the chorion leads to the trilaminar trophoblast cell structure in the placental labyrinth. Development. 2008. https://doi.org/10.1242/dev.020099.

17. Rossant J, Cross JC. Placental development: lessons from mouse mutants. Nat Rev Genet. 2001. https://doi.org/10.1038/35080570.

18. Pijnenborg R. The guide to investigation of mouse pregnancy. Placenta. 2015. https://doi.org/10.1016/j.placenta.2014.11.023.

19. Zhang Y, et al. Dynamic epigenomic landscapes during early lineage specification in mouse embryos. Nat Genet. 2018. https://doi. org/10.1038/s41588-017-0003-X

20. Hemberger M, Hanna CW, Dean W. Mechanisms of early placental development in mouse and humans. Nature Rev Genet. 2020;21:27-43. Hemberger et al. recognized for its importance as a recent review of mouse placental development that the authors recommend.

21. Smith ZD, et al. Epigenetic restriction of extraembryonic lineages mirrors the somatic transition to cancer. Nature. 2017. https://doi. org/10.1038/nature23891

22. Auclair G, Guibert S, Bender A, Weber M. Ontogeny of $\mathrm{CpG}$ island methylation and specificity of DNMT3 methyltransferases during embryonic development in the mouse. Genome Biol. 2014. https://doi.org/10.1186/s13059-014-0545-5.

23. Legault LM, et al. Developmental genome-wide DNA methylation asymmetry between mouse placenta and embryo. Epigenetics. 2020. https://doi.org/10.1080/15592294.2020.1722922.

24. Decato BE, Lopez-Tello J, Sferruzzi-Perri AN, Smith AD, Dean MD. DNA methylation divergence and tissue specialization in the developing mouse placenta. Mol Biol Evol. 2017. https://doi.org/ 10.1093/molbev/msx112.

25. Jagannathan-Bogdan M, Zon LI. Hematopoiesis Dev. 2013;140:2463-7.

26. Wu Y, Hirschi KK. Regulation of hemogenic endothelial cell development and function. 2020. https://doi.org/10.1146/annurevphysiol-021119.

27. Hirschi KK. Hemogenic endothelium during development and beyond. Blood. 2012;119:4823-37.

28. Fang JS, Gritz EC, Marcelo KL, Hirschi KK. Isolation of murine embryonic hemogenic endothelial cells. J Vis Exp. 2016;2016.

29. Ottersbach K, Dzierzak E. The placenta as a haematopoietic organ. Int J Dev Biol. 2010;54:1099-106.

30. Gekas C, Dieterlen-Lièvre F, Orkin SH, Mikkola HKA. The placenta is a niche for hematopoietic stem cells. Dev Cell. 2005;8:365-75.

31. Coşxkun $\mathrm{S}$, et al. Development of the fetal bone marrow niche and regulation of HSC quiescence and homing ability by emerging osteolineage cells. Cell Rep. 2014;9:581-90.
32. Gritz E, Hirschi KK. Specification and function of hemogenic endothelium during embryogenesis. Cell Mol Life Sci. 2016;73:1547-67.

33. Alberts B, Johnson A, Lewis J. Blood vessels and endothelial cells new endothelial cells are generated by simple duplication of existing. Mol Biol Cell 4th Ed. 2002;4126:24-28.

34. Sasaki T, et al. Regulation of hematopoietic cell clusters in the placental niche through SCF/Kit signaling in embryonic mouse. Development. 2010. https://doi.org/10.1242/dev.051359.

35. Rhodes KE, et al. The emergence of hematopoietic stem cells is initiated in the placental vasculature in the absence of circulation. Cell Stem Cell. 2008. https://doi.org/10.1016/j.stem.2008.01.001.

36. Ottersbach K, Dzierzak E. The murine placenta contains hematopoietic stem cells within the vascular labyrinth region. Dev Cell. 2005. https://doi.org/10.1016/j.devcel.2005.02.001.

37. Corbel C, Salaün J, Belo-Diabangouaya P, Dieterlen-Lièvre F. Hematopoietic potential of the pre-fusion allantois. Dev Biol. 2007. https://doi.org/10.1016/j.ydbio.2006.08.069.

38. Zeigler BM, et al. The allantois and chorion, when isolated before circulation or chorio-allantoic fusion, have hematopoietic potential. Development. 2006;133:4183-92.

39. Liu Y, et al. Single-cell RNA-seq reveals the diversity of trophoblast subtypes and patterns of differentiation in the human placenta. Cell Res. 2018;28:819-32.

40. Nuschke A. Activity of mesenchymal stem cells in therapies for chronic skin wound healing. Organogenesis. 2014;10:29-37.

41. Parolini $\mathrm{O}$ et al. Concise Review: Isolation solation and characterization of cells from human term placenta: outcome of the first international workshop on placenta derived stem cells. Stem Cells. 2008;26.

42. Dominici $\mathrm{M}$ et al. Minimal criteria for defining multipotent mesenchymal stromal cells. The International Society for Cellular Therapy position statement. Cytotherapy. 2006;8: 315-317.

43. Papait A, et al. Mesenchymal stromal cells from fetal and maternal placenta possess key similarities and differences: potential implications for their applications in regenerative medicine. Cells. 2020. https://doi.org/10.3390/cells9010127.

44. Murphy SP, Choi JC, Holtz R. Regulation of major histocompatibility complex class II gene expression in trophoblast cells. Reprod Biol Endocrinol. 2004;2:1-8.

45. Scialdone A et al. Resolving early mesoderm diversification through single-cell expression profiling. Nature. 2016;535:289293. (Scialdone et al. is recognized for its importance as the first mouse placenta scRNA-seq study to conduct integrated analysis across multiple developmental stages.)

46. Marsh B, Blelloch R. Single nuclei RNA-seq of mouse placental labyrinth development. Elife. 2020;9:1-27. Marsh \& Blelloch $\mathbf{2 0 2 0}$ is recognized for its importance as the first mouse placenta snRNA-seq study and the first study to investigate multiple stages including the fully developed placenta (E9.5-E14.5) at the single cell level.

47. Zhu D, Gong X, Miao L, Fang J, Zhang J. Efficient induction of syncytiotrophoblast layer II cells from trophoblast stem cells by canonical Wnt signaling activation. Stem Cell Rep. 2017;9.

48. Ibarra-Soria X et al. Defining murine organogenesis at single-cell resolution reveals a role for the leukotriene pathway in regulating blood progenitor formation. Nat Cell Biol. 2018;20:127-134. Ibarra-Soria et al. is recognized for its importance as the first mouse placenta scRNA-seq to apply 10X Genomics.

49. Ware CB, Horowitz MC, Renshaw BR, Hunt JS, Liggitt D, Koblar SA, Gliniak BC, McKenna HJ, PT TB. Targeted disruption of the low-affinity leukemia inhibitory factor receptor gene causes placental, skeletal, neural and metabolic defects and results in perinatal death. Development. 1995;121:1283-1299.

50. Li H, Huang Q, Liu Y, Garmire LX. Single cell transcriptome research in human placenta. Reproduction. 2020;160:R155-67. 
51. Turco MY, Moffett A. Development of the human placenta. Development (Cambridge). 2019;146 (2019). Turco \& Moffet 2019 is recognized for its importance as a recent review of human placental development that the authors recommend.

52. Xiao Z, Yan L, Liang X, Wang H. Progress in deciphering trophoblast cell differentiation during human placentation. Curr Opin Cell Biol. 2020;67:86-91.

53. Pavličev M, et al. Single-cell transcriptomics of the human placenta: Inferring the cell communication network of the maternalfetal interface. Genome Res. 2017;27:349-61.

54. Chang WL et al. PLAC8, a new marker for human interstitial extravillous trophoblast cells, promotes their invasion and migration. Development. 2018;145.

55. Li M, Chen L, Zhang J, Xiong C, Li X. The SARS-CoV-2 receptor ACE2 expression of maternal-fetal interface and fetal organs by single-cell transcriptome study. PLoS One. 2020;15.
56. Pique-Regi R, et al. Does the human placenta express the canonical cell entry mediators for sars-cov-2? Elife. 2020;9:1-15.

57. Lü M, Qiu L, Jia G, Guo R, Leng Q. Single-cell expression profiles of ACE2 and TMPRSS2 reveals potential vertical transmission and fetus infection of SARS-CoV-2. Aging (Albany. NY). 2020. https://doi.org/10.18632/aging.104015.

58. Cui D, et al. Single-cell RNA expression profiling of SARS-CoV2-related ACE2 and TMPRSS2 in human trophectoderm and placenta. Ultrasound Obstet Gynecol. 2021. https://doi.org/10.1002/ uog. 22186.

59. Colson A, et al. COVID-19 during pregnancy: clinical and in vitro evidence against placenta infection at term by SARS-CoV-2. Am J Pathol. 2021. https://doi.org/10.1016/j.ajpath.2021.05.009.

Publisher's Note Springer Nature remains neutral with regard to jurisdictional claims in published maps and institutional affiliations. 